SYRACUSE UNIVERSITY

Progress Report on

"RESEARCH PROGRAM IN ELEMENTARY PARTICLE THEORY"

\title{
Supported by
}

U. S. Energy Research and Development Administration

Under Contract No. E(11-1)-3533

and by

Syracuse University

For the period ending August 31, 1976 


\section{DISCLAIMER}

This report was prepared as an account of work sponsored by an agency of the United States Government. Neither the United States Government nor any agency Thereof, nor any of their employees, makes any warranty, express or implied, or assumes any legal liability or responsibility for the accuracy, completeness, or usefulness of any information, apparatus, product, or process disclosed, or represents that its use would not infringe privately owned rights. Reference herein to any specific commercial product, process, or service by trade name, trademark, manufacturer, or otherwise does not necessarily constitute or imply its endorsement, recommendation, or favoring by the United States Government or any agency thereof. The views and opinions of authors expressed herein do not necessarily state or reflect those of the United States Government or any agency thereof. 


\section{DISCLAIMER}

Portions of this document may be illegible in electronic image products. Images are produced from the best available original document. 


\section{PRE AMB LE}

This has been an active year for the Syracuse High Energy Theory group. Research on the long standing interests of the group members has continued and in addition several interesting new results, possibly opening up new fields, have emerged.

The high energy behavior of form factors in non-Abelian gauge theories was investigated using a diagrammatic technique and some advances in the analysis of complicated diagrams were made. ${ }^{1,2}$ Chiral symmetry for the charmed particles was studied and the possibility of an asymmetric vacuum with significant consequences for their weak decays was noted. ${ }^{3,4}$ A very detailed investigation of charmed particle weak decays was carried out using the vector dominance mode1. 5 The dispersion theoretic bootstrap approach to charmed baryon resonances was set up. ${ }^{6}$ The long standing problem of charge-monopole scattering ${ }^{\circ}$ in quantum mechanics was solved using a functional technique. It was found that the scattering amplitude was not an analytic function of the scattering angle and that the classical result was not obtained for $\hbar \rightarrow 0$. Several studies bearing on the theory of the 't Hooft monopole were carried out. ${ }^{7,8} \mathrm{~A}$ possible relevance of the ' $t$ Hooft monopole to certain structures in ${ }^{3} \mathrm{He}$ was noticed and studied. ${ }^{9,10,11} \mathrm{~A}$ unified model of strong interactions and gravity which may lead to quark confinement as well as renormalizability was proposed. ${ }^{12,13}$ The formulation of spontaneously broken supersymmetric gauge theories was investigated ${ }^{14}$. Finally, a method was devised for carrying out an exact integration 
of the Feynman path integral so that the quantum mechanical Green's function could be expressed explicitly in terms of dunctions obtained from lassical mechanics for one dimensional systems. ${ }^{15}$

$\therefore$ Besides the activities above; which are discussed in more detail below, members of the theory group attended topical and national meetings and visited other institutions to give seminars and colloquia. Informal high energy seminars were held sometimes jointly with the Experimental High Energy group at Syracuse. Theorists from other institutions were invited to present more formal seminars concerning their work.

K.C. Wali spent the fall semester on sabbatical leave at the Institut des Hautes Etudes Scientifiques, Bures-sur-Yvette, France. R. Cahalan visited the National Academy of Science and Polska Akademia Nauk, Institute of Theoretical Physics, Warsaw, Poland as an exchange scientist for six months. He will be completing his third year with our group. His plans for the next year are uncertain as yet. Stephen Blaha, who has contributed very actively to our group, will be leaving Syracuse. He has accepted a position at Williams College. Three students, G. Campbe11, Jr., D. Knight and $\mathrm{J}$. Kandaswamy are well on their way to completing their. $\mathrm{Ph} . \mathrm{D}$. requirements. 


\section{RESEARCH REPORT}

The recent discoveries of new particles $\left(J / \psi(3 \cdot 1), \psi^{\prime}(3 \cdot 7)\right.$, $\left.\psi^{\prime \prime}(4 \cdot 1), \ldots\right)$ and other related phenomena have ushered high energy physics into a new era. While the experimental situation in these new areas is yet to become clear, there are several theoretical ideas which need to be investigated. The syracuse theory group in the past year has actively participated in an understanding of the new physics and its theoretical implications. It also has continued to make progress in some other long standing projects. In what follows we shall summarize the main results of these research efforts. I. Non-Abelian Gauge Theories

In the last year's progress report, ${ }^{1}$ we had described in some detail the motivation for studying the Non-Abe1ian Gauge theories and especially the asymptotic behaviour of the form factors. Cahalan (also Cornwall and Tiktopoulous) have shown that the infrared logarithms up to sixth order add up to give the appropriate expansion of an exponential form analogous to the situation in the usual quantum electrodynamics. It is an extremely important and at the same time a difficult problem to generalize this result to all orders. The first step in this direction is to clarify the topology of the leading graphs in a given Yang-Mills gauge theory. As yet, however, one does not know the appropriate gauge theory which describes all interactions in a unified manner. In any gauge theory, the form factors will be given by the sum of products of two factors - one 
that depends on the specific gauge group and the other on the momentum space. Also, in any gauge theory, in order to produce the desired exponential form, there have to be delicate concellations between higher order and lower order contributions.

Cahalan and Knight ${ }^{2}$ have considered the group theory part of the problem for the $U_{N}$ scalar form factor. G.'t Hooft has shown that if one considers the fundamental representation of $U_{\mathrm{N}}$ and keeps only the leading power of $\mathrm{N}$ in each order of the coupling constant, the group factor is determined only by the number of "index loops". The momentum factors can then be directly computed. Such a procedure provides a much simplified context in which to check results which are expected to apply to any gauge group. Cahalan and Knight give a simple explicit and iterative prescription for constructing a11 the relevant planar graphs (i.e. graphs which give leading asymptotic behaviour) in the 't Hooft approximation. In this paper they also show that the planar results for $U_{N}$ also holds for $S_{N}$. It is hoped that their work combined with the appropriate momentum space factors will lead to a gauge invariant generating functional for the above mentioned graphs.

II. Chiral Symmetry, Weak Interactions and the New Particles

The study of various aspects of chiral symmetry has been one of the on-going projects at Syracuse. Schechter and Singer $^{3}$ and Schechter, Singer and Kandaswamy ${ }^{4}$ have extended the SU(3) o-model to SU(4) to include the effect of the new quantum number 'charm'. The main idea behind this program is to understand the mass spectrum 
of the spin zero particles and the decays based on a symmetry breaking interaction which transforms like a linear combination of quark mass terms. In the framework of a general linear $\sigma$-mode 1 , it is relatively easy to include the effects of vacuum symmetry breaking. They have shown that the charmonium picture can be naturally accomodated if $\frac{\mathrm{m}_{\mathrm{c}}}{\mathrm{m}_{\mathrm{s}}} \simeq \frac{\mathrm{m}_{\mathrm{s}}}{\mathrm{m}_{\mathrm{u}}} \simeq 30$, where the $\mathrm{m}$ 's denote appropriate "quark masses". An amusing feature 4 , different from the SU(3) case is that the $\mathrm{SU}(4)$ invariance of the vacuum is broken to a nonnegligible extent when one imposes the condition of renormalizability on the $\sigma$-mode 1 . A direct consequence is the enhancement of the pure leptonic decay modes of the charmed pseudoscalars. If heavy leptons with masses less than those of the charmed pseudoscalars exist (as experiments at SLAC seem to indicate), this will lead to important consequences for the analyses of the dimuon events. So far the analyses that have been carried out disregard this possibility. It. is also to be noted that the enhancement of the single particle matrix elements of the charm-changing axial vector currents (which lead to leptonic decays) does not have any important effect on the main non-leptonic decay modes in the simplest version of such an SU(4) mode1.

III. SU(4) Symmetry and New Particles

As is well known, the most popular interpretation of $\psi$ and ' $\psi^{\prime}$ is based on a new kind of quark (charmed quark). in addition to the usual triplet of quarks. This naturally leads to the consideration of su(4) symmetry as a symmetry of strong interactions. Borchardt 
(along with Mathur and Okubo of the University of Rochester) have in the past studied the masses and the prominent decay modes of the new particles (particles with charm quantum number or charmed particles) which accompany the known hadrons in low lying hadronic multiplets. Since then extensive experimental search has failed to produce direct evidence for the existence of the new particles. Borchardt ${ }^{5}$ (along with Mathur) suggests that the lowest lying charmed particles are vector mesons (and not pseudoscalar mesons as commonly assumed). They use the vector dominance model to calculate the (quasi-) two body rates of the charmed vector mesons and show how one can explain the small branching ratio into 2 or 3 pseudoscalar mesons; they suggest looking for 4-body final states, that is the final states involving the quasi-two body decays of two vector mesons such as $\rho, K^{*}, \phi$ etc. They single out $K^{*} \rho+K \pi \pi \pi$ as the channel which is most promising for charm search.

Campbell is investigating a dynamical approach to the baryonic resonances in $S U(4)$. The $J^{P}=\frac{3^{\dagger}}{2}$ decuplet of SU(3) goes over to a 20-plet resonances in $\mathrm{SU}(4)$. The stable baryon $\mathrm{J}^{\mathrm{P}}=\frac{1}{2}^{\dagger}$ octet and the pseudoscalar meson octer go over to another 20-plet and 15-plet of SU(4). The Chew-Low reciprocal bootstrap mechanism has been very successful in explaining several features of the baryonic multiplets in SU(3) symmetry. Campbe11 has extended these considerations to SU(4) symmetry. He has constructed ${ }^{6}$ the necessary crossing matrix for meson-baryon scattering and has shown that the 20-plet baryon exchange forces do indeed produce strong attractive forces in the desired channel which contains the SU(3) decuplet. Currently he 
is calculating the masses and widths of these resonances using a dispersion-theoretic $N / D$ method.

IV. Monopoles

a) Abelian Theories

The interest in monopole type theories stems from many reasons ${ }^{7}$. Consequently for the past few years, it has occupied the interests of several members of our group. A well-known problem in the treatment of systems containing monopoles is that most approximation schemes violate rotational invariance and therefore lead to meaningless results. During the last year, Balachandran, Borchardt, Cahalan, Chang and Stern, along with Rupertsberger at the University of Vienna, have studied alternative approaches to overcome this problem. They have proved that approximation schemes of the semiclassical type are consistent with rotational invariance. The chargè-monopole scattering amplitude in non-relativistic quantum mechanics has been worked out in detail in this approximation. Several remarkable features of such systems have emerged in the course of the investigation. Thus the quantum cross-sections do not reduce to classical ones as $h \rightarrow 0$. The absence of a classical limit in the normal sense throws doubt on many recent classical considerations on monopoles. A1so, at least in the preceding approximation, the quantum scattering amplitude is not an analytic function of scattering angle at all. If this feature persists in the field theoretic case, it will clearly have important consequences for S-matrix type theories based on analyticity and unitarity. 
As important side results of the above investigation, Balachandran and Rupertsberger have succeeded in streamlining the calculations of Dashen, Hasslacher and Neveu on semi-classical approximations to functional integral representations of Green's functions with velocity independent interactions, and generalizing the methods to arbitrary Hamiltonians.

They are in the process of settling some remaining relatively minor points concerning the monopole-charge scattering amplitudes. Once this is accomplished, the results will be written up in a series of papers.

a) Non-Abelian Theories

Recently G: 't Hooft has constructed a model for monopoles starting from the Yang-Mills Lagrangian supplemented by Higgs fields. Considering only the asymptotic solutions to the resulting nonlinear equations, he was able to make plausible arguments for the existence of a monopole in the theory. Last year Prasad and Sommerfield gave exact solutions to a special case of these equations. A remarkable feature of these solutions is that they are regular everywhere. As an aid to understanding the 't Hooft solution, Schechter has investigated ${ }^{8}$ the classical motion of a Yang-Mills test particle in this field. A convenient parameterization for the particle's isospin vector enabled him to see that the test particle moves as a charge in a pure monopole field only at large distances. At short distances, the motion is different from that expected from pure monopole field. He has found an exact asymptotic solution 
for the motion of the particle's iso-spin vector and discussed its physical interpretation.

Wali (along with Miche 1 and 0 'Raifeartaigh) have been concerned with the possible generalizations of the 't Hooft monopole. Under the assumption that the solutions have a factorized form (radial part times angular part) in which the internal symmetry indices are carried by the angular part, they have obtained interesting restrictions on the functions which provide the solutions to the generalized non-linear equations that arise. What is not clear at the moment is the exact nature or type of monopoles in different type of solutions.

A relatedbut different line of investigation in which Balachandran, Borchardt and Stern have made progress during the course of this year concerns theories of point particles coupled to non-Abelian gauge fields. S.Y.Wong has written down the classical equations of such systems. Balachandran et al., have solved the non-trivial problem of finding a Lagrangian for such systems. In the coming months, they hope to set up the canonical formalism for these systems, develop their quantum mechanics and study their various properties. Hopefully, such a study will clarify current conjectures regarding confinement mechanisms in non-Abelian gauge theories. V. Topological Structures and the 't Hooft Monopole

The 't Hooft monopole discussed in the previous section is topological in nature rather than dynamical, that is, the quantization of the conserved magnetic charge does not depend on the 
dynamical evolution of the fields, but only on their topological nature. Topological quantum numbers are related to homotopy theory. Blaha has studied in detail this relation and used topological considerations in a novel way. ${ }^{9,10}$ He has shown that an analogue of the magnetic monopole exists in the A phase of superfluid ${ }^{3} \mathrm{He}$. He predicts new structures called vortons which are point like singularities to which vortices are attached. The vortices in the circulation of ${ }^{3} \mathrm{He}-\mathrm{A}$ become completely analogous to Dirac string in the monopole theory. Besides the inherent interest of such structures in ${ }^{3} \mathrm{He}-\mathrm{A}$ theory, the existence of vortons enhances the expectation that true magnetic monopoles occur in nature - if one believes nature repeats its tricks. Furthermore, a more detailed analogy can be made between the vortons and the 't Hooft model of the magnetic monopole. In this analogy the Higgs field is associated with the local orbital angular momentum of the ${ }^{3} \mathrm{He}$ Cooper pairs while the Yang-Mills field is associated with the Landau-Ginzberg order parameter. Thus the program initiated by Nielsen and olesen, who identified the string structure of an Abelian Higgs model with the vortex structure of a Landau-Ginzberg model of superconductivity, is now completed by the identification of the monopole structure of a nonAbelian Higgs model with the vorton structure in the Landau-Ginzberg. mode 1 of superfluid ${ }^{3} \mathrm{He}-\mathrm{A}$.

Blaha has also shown ${ }^{11}$ that the order parameter in the LandauGinzberg theory for ${ }^{3} \mathrm{He}$ can be regarded as a complex Yang-Milis field. If the dipolar forces and external fields are neglected, the order parameter is complex and has tensorial properties, 
transforming like a vector under independent rotations in spin space and in coordinate space. The consequence is that the free energy in the Landau-Ginzberg region has the form of a massive Yang-Mills Lagrangian. Thus one has a physical system to study the dynamics of complex Yang-Mills fields which have been discussed recently by Yang and Wu.

VI. Unified Gauge Mode1s of Interactions

The successes of the Salam-Weinberg model in providing a unified description of weak and electromagnetic interactions has given rise to intensive efforts to describe all interactions in a unified manner within the framework of renormalizable quantum field theory. A set of local 'quark' fields (elementary spin $1 / 2$ Fermi fields) coupled to an octet of colored gluons (gauge mesons) a $1 \mathrm{a}$ Yang and Mills, is supposed to produce strong interactions. The infrared divergences present in the theory are expected to confine the quarks within the observable hadrons (which are all color singlets). However, there is no convincing dynamical theory in the four-dimensional world which produces such a quark confinement. A slightly different and unconventional approach has been proposed by $\mathrm{Blaha}^{12}$, who introduces fourth order derivative terms in the color gluon equations. He has shown that the resulting second quantized theory has a natural mechanism for quark confinement. The theory has also other virtues and the naive questions that one may raise based on unitarity and causality, can be satisfactorily answered.

Looking for further justification of the fourth order derivative 
terms, he has studied quantum field theories describing gravitational interactions. Such theories are in general non-renormalizable and in some versions of such theories, the simplest way of obtaining at least power counting renormalizability appears to be the introduction of fourth order derivative terms. Starting from this observation and the presence of some formal similarities between gravitation and vector field theories which have confinement, he has constructed a model unifying strong and gravitational interactions. 13 The model in the strong interaction sector reduces almost exactly to that of reference 12 . Thus it has quark confinement and ultraviolet free behaviour (asymptotic freedom). In the gravitational sector it is renormalizable at short distances and goes over into the Einstein model at large distances. Taking account of the value of the gravitational coupling constant and the energy at which Bjorken scaling appears ị deep inelastic lepton-nucleon scattering, he can find the value of the coupling constants in the model. Interestingly, the Fermi weak coupling constant appears as a simple algebraic ratio of strong coupling constants in the model. Furthermore, Fermi-like interaction terms naturally appear in the model. Thus it is possible that the weak interactions may also be introduced in the model in a natural way.

Recently a radically different approach to unify all interactions has been proposed by Arnowitt and Nath within the framework of supersymmetry schemes. They would like to regard all particles (not only the conventional $\mathrm{J}^{\mathrm{P}}=1^{-}$gauge particles) as gauge particles 
and construct a Lagrangian in which all the interactions are determined by a common non-Abelian gauge invariance. This approach is termed 'gauge supersymmetry'.

In the framework of such a gauge supersymmetry, the fundamental metric tensor superfield $g_{A B}\left(x^{\mu}, \theta^{\alpha}\right.$ ) (where $x^{\mu}$ are space-time coordinates and $\theta^{\alpha}$ anti-commuting $c$-numbers) is assumed to contain large arrays of physical fields. A spontaneous symmetry breakdown occurs if some fields in $g_{A B}$ can assume non-vanishing vacuum expectation values. The "vacuum metric" $g_{A B}(0)\left(\theta^{\alpha}\right)$ should satisfy the general field equation proposed; i.e. the Ricci tensor calculated from $g_{A B}{ }^{(0)}\left(\theta^{\alpha}\right)$ alone must obey the identity $R_{A B}{ }^{(0)}=\lambda g_{A B}(0)$. Previousiy no such kind of vacuum metric was found. Chang has shown the existence of a $g_{A B}(0)$ which satisfies the proposed field equation. 14 The vacuum metric thus found is gauge invariant under the conventional supersymmetry transformations. Once such a vacuum metric is found, one can separate the fundamental metric into two parts: $g_{A B}=g_{A B}(0)+h{ }_{A B}$. and rewrite the Lagrangian in terms of $g_{A B}(0)$ and $h_{A B}$. This is of great interest because the new "effective Lagrangian" can show how the "Higgs mechanism" works and masses of some particles arise. Following a scheme initiated by Arnowitt and Nath, Chang has rewritten the effective Lagrangian density in such a desired form, up to all orders in $h_{A B}$ expansion. This work will be useful for further studies of spontaneous breakdown in gauge supersymmetry. VII. Exact Integration on Feynman Path Integrals As mentioned earlier (Section IV), the study of monopole theories 
has led several members of the group to the investigation of quantization methods, Feynman's path integral approach to quantum mechanics has received a great deal of interest recently since it has proved very useful in quantizing Yang-Mills type theories using the functional methods. In this connection Chang has proposed ${ }^{15}$ a new technique for integrating phase space path integrals. He has introduced the concept of "path bundles" and considered the path integral as an infinite sum of (const) $x \exp \left\{\frac{i}{h}\right.$ Action $\}$ over a complete set of path bundies. Each bundle is assumed to possess widths differentiable in time. Using Hamilton's principal function $S(q, P, t)$ as a gener ating function for canonical transformations, he has been able to determine how the path integral changes under canonical transformations. Especially, it is found that the integrator measure $\mathcal{O}_{\mathrm{q}} \mathcal{C H}_{\mathrm{p}}$ changes into $\mathcal{O}_{\mathrm{Q}} \hat{L}_{\mathrm{P}} \times\left[\mathrm{D}(\mathrm{q}, \mathrm{P}, \mathrm{t}) \mathrm{D}\left(\mathrm{q}_{\mathrm{o}}, \mathrm{P}_{0}, \mathrm{t}_{0}\right)\right]^{1 / 2}$, where $\mathrm{D}(\mathrm{q}, \mathrm{P}, \mathrm{t}) \equiv$. $\operatorname{det}\left(\frac{\partial^{2} S}{\partial q \partial P}\right) q, P, t$. This result differs from naive change of variables $\alpha Q_{\mathrm{q}} \mathscr{L}_{\mathrm{p} \rightarrow \mathcal{L} \mathrm{Q}} \mathscr{C}_{\mathrm{p}}$. More striking1y, the integrations on

$$
\mathcal{L Q}_{Q} \mathcal{L}_{\mathrm{P}}^{\mathrm{C}} \equiv_{\mathrm{j}=1}^{\mathrm{N}-1} \mathrm{dQ}_{\mathrm{j}} \prod_{\mathrm{s}=1 / 2}^{\mathrm{N}-1 / 2} \mathrm{dP}_{\mathrm{s}} \text { happen to be very simple, }
$$

because each integration on $Q_{j}$ yields a delta function $\delta\left(P_{j+1 / 2}-P_{j-1 / 2}\right)$. The complicated path integral can thus be integrated into a single ordinary integral. From the integrated path integral one can obtain the exact Green's function for a harmonic oscillator. For a regular one dimensional Hamiltonian where $H=p^{2} / 2 m+V(q)$, he has shown that the integrated path integral fulfills the initial condition and Schroedinger equation appropriate to the corresponding quantum mechanicaI Green's function. 


\section{Concluding Remarks}

We have summarized qualitatively and rather briefly some of the main areas of research and results obtained so far. It should be clear that the group as a whole has contributed significantly to several areas of great current interest in particle theory. 


\section{References}

1. Progress report on "Research Program in Elementary Particle Theory". Submitted to the U.S. Energy Research and Development Administration, May 23, 1975. (C00-58)

2. Construction of Planar Diagrams; R. Cahalan and D. Knight, submitted to Pliys. Rev. D, May 1976. (COO-57)

3. SU(4) Sigma Mode 1; J.Schechter and M.Singer, Phys. Re $\bar{v} \cdot \underline{D} 12$, 2781 (1975): ( $00-60)$

4. Possible Enhancement of the Leptonic Decays of Charmed Pseudoscalars; J.Kandaswamy, J.Schechter and M.Singer. to be published in Phys. Rev.:D: $(\mathrm{COO}-66)$

5. Weak Decay Modes of Charmed Mesons; S.Borchardt and V.S.Mathur; (University of Rochester preprint) accepted for publication in Phys. Rev. Letters. 1976

6. Baryon Exchange Forces in SU(4) Symmetry; George Campbe11, Jr. Phys. Rev. D13, 662 (1976).

7. See for instance:

Hamiltonian Formulation of Monopole Theories with Strings; A.P. Balachandran, R. Ramachandran, H.Rupertsberger, J.Schechter and K.C.Wali; Phys. Rev. D13, 354 (1976).

Strings, Monopoles and Meson States; with same authors. Phys. Rev. D13, 361 (1.976).

8. Yang Mills Particle in 't Hooft's Field; J.Schechter, submitted to Phys. Rev. April (1976). (C00-71)

9. Quantization Rules for Point Singularities in Superfluid ${ }^{3} \mathrm{He}$; S.Blaha, Phys. Rev. Letts., to be published. (C00-65).

10. An Experimentally Accessible Analog of the Magnetic Monopole in ${ }^{3} \mathrm{He}-\mathrm{A}$. S.Blaha, submitted to Phys. Rev. Letts. (COO-69)

11. Yang Mills Approach to the Superfluid ${ }^{3}$ He Free Energy; S.Blaha, Submitted to Phys. Rev. Letts. ( $000-70)$

12. A Second Quantized Non-Abelian Field Theory for Hadrons with Quark Confinement and Scaling Deep Inelastic Structure Functions; Phys. Rev. D11, 2921 (1975). S.Blaha, author.

13. Quantum Gravity and Quark Confinement; S.Blaha, submitted to Phys. Rev. (1976) (C00-68) 
14. Effective Lagrangian Density in Gauge Symmetry. S.S.Chang, Submitted to Phys.Rev. ( $\mathrm{COO}-67)$

15. Exact Integration on Feynman Path Integrals; S.S.Chang, submitted to Phys.Rev. (COO-72) 


\title{
APPENDIX A
}

ERDA Reports by the Scientific Staff Under Contract No. E(11-1)-3533

\begin{abstract}
C00-3533-58. Progress Report on "Research Program in Elementary
Particle Theory". Submitted to the U.S. Energy

Research and Development Administration, May 23,; 1975.

C00-3533-59 On the Multi Regge Behavior of a certain Class of Dual

Resonance Models; P.H.Frampton and Dan Knight. Submitted to I1 Nuovo Cimento October, 1975.

C00-3533-60 SU(4) Sigma Mode1; J.Schechter and M.Singer, Phys. Rev.D12, 2781 (1975).

C00-3533-61 Current Status of Baryon Spectroscopy; K.C.Wa1i, Ta1k published in "New Direction of Hadron Spectroscopy" proceedings of the International Symposium, Argonne National Laboratory, Argonne, I11. (July 1975).

C00-3533-62 Infrared Behavior of the Reggeon Field Theory for the Pomeron. W.Bardeen, J.W.Dash, S.Pinsky and V.Rab 1. Phys. Rev. D12, 1820 (1975).
\end{abstract}

C00-3533-63 Baryon Exchange Forces in SU(4) Symmetry; George Campbel1. Phys. Rev. D13, 662 (1976).

Co0-3533-64 Proceedings of the Fourth International Symposium on $\bar{N} N$ Interactions, May 2-4, 1975. Editors,. T.E.Kalogeropoulos and K.C.Wali. Syracuse University, Syracuse, N.Y.

C00-3533-65 Quantization Rules for Point Singularities in Superfluid $3 \mathrm{He}$; S.Blaha, Phys. Rev. Letts. to be published, (1976).

C00-3533-66 Possible Enhancement of the Leptonic Decays of Charmed Pseudoscalars. J.Kandaswamy, J.Schechter and M.Singer, Phys. Rev. D. to be published (1976).

C00-3533-67 Effective Lagrangian Density in Gauge Symmetry. S.S.Chang, Submitted to Physical Review (1976).

C00-3533-68 Quantum Gravity and Quark Confinement; S.Blaha, Submitted to Physical Review (1976).

C00-3533-69 An Experimentaily Accessible Analog of the Magnetic

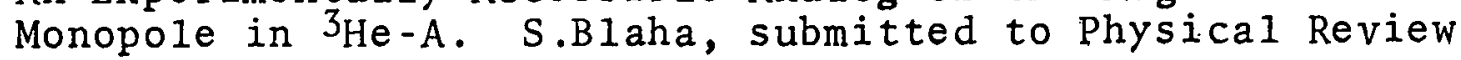
Letters . (1976) 

C00-3533-70 Yang Mills Approach to the Superfluid ${ }^{3}$ He Free Energy, S.BIaha, Submitted to Physical Review Letters, April (1976).

COO-3533-71 Yang Mills Particle in 't Hooft's Field. J.Schechter, submitted to Phys. Rev. April (1976).

C00-3533-72 Exact.Integration on Feynman Path Integrals; S.S.Chang, Submitted to Phys. Rev. April (1976).

$\begin{array}{ll}* & \text { Construction of Planar Diagrams; R.Cahalan and D.Knight, } \\ \text { to be submitted, May }(1976) .\end{array}$ 
1. Sonce Eunaris on the Masses of Elementary Particles; Proceedings of the Cosis ic Ray Symposjum at Ahmedabad, India (136(1).

A Note on Scattcring and Production Amplitudes; Nuclear Physics $1 S$, 81(1960); with N. R. Ranganatinan.

3. A Note on K-Hyperon Relative Parities; Nuovo Cimento 16, $1142(1260)$; with.N. R. Ranganthan.

4. On a Mode1 for E-Decars; Nuovo Cimento 18, 1269(1960); with N. R. Ranganthan.

5. Tests for Compound Models; Nuovo Cimento 19, 190(1961); with G. Bhanathi, S. Indumathi and $N$. G: Deshpande.

6. On a Isotopic Spin Scheme for Leptons; Proc. Ind. Acad. Sc. 53, 1(2061); with $A$. Ramakrishnan and $R$. N. Ranganathan.

7. On an Isobaric Spin Scheme for Leptons and the Leptonic Decays of: Strange Particles; Nuclear Physics 26, 52(1961); with $\therefore$. G. Desingunde.

8. Some Remarks on Sakurai's Theory of Strong Interactions; Nuovo Cimento 20, 1022(1961); with N. G. Deshfande.

9. A Note on the Non-leptonic Decay Modes of the $\Xi$ Hyperon; Prog. Theoretical Phys. 26, 792 (1961); with K. Venkatesan.

10. On the Value of the Axial Vector Renormalization in B-Decay; Nuoro Cimento 23, $428(1962)$.

11. The K-meson Pion Scattering; Nuovo Cimento 23, 402(1962).

12. Low Energy $\mathrm{K}^{+}$Nucleon Scattering; Nuovo Cimento 24, $369(1.962)$; with A. Ramakrishnan and K. Raman.

13. Partjal-lVave Dispersion Relations $\Lambda-N$ Scattering; Nuovo Cimento 24, 980(1902); with A. Ramakrishnan.

14. On the $Y^{*}$-resonances; Nuovo Cimento 24, 1006(1962); with A. Ranakishar T. K. Rädha and R. Thunga.

-15. Photoproduction of Pions on $\Lambda$-hyperon; Nuovo Cimento 25, 939 (1962); witi $A$. Ramekrishnar, $T$. K. Radha and $R$. Thunga.

16: On the Spin and Parity of the $Y^{*}$-resonances; Nuovo Cinento 25, 723(1962.j; with A. Ranak:ishnan, T. K. Radha and R. Thunga.

17. On a Possible $\equiv-\pi$ Rescnance; Phys. Letters 2, 126(1962); with H. Pietschanan.

Sumenregcln für Nukleon Formfaktoren; Acta Physics Austriaca 1e, 362(1962); with H. pieischinann. 
19. On the rsovector Forn lictors of the Nuclecn; Nuclear Physics 13, 32].(1903); with. H. Pieischinann.

Tine Present Status of the New Resonances; Acta Physica Austriaca de, $159(3.963)$; with H. Pietschmann.

21. Uniqueness of the Parial-llave Mmplitude; Phys. Rev. 132, $894(196 \vdots)$.

22. Ccrtain Questions of Consistency in S-matrix Theory; Fhys. Rev. 133, B68 $(1.961)$.

23. Boundary Conditions for a lartial-wave Mmplitude; J: Math. Phys. 5, 614 (1964).

21. Sonc Consequences of the Isospin Commutation Relations for Isovector Form Factors; Ann. Phys. 29, 161(1964); with H. Pietschmann and W. Kumner.

25. Some Inequalities for the Forward Scattering Amplitude; Phys. Rev. 131, $13197(1964)$.

26. Asymptotic Bchavjor of Electromagnetic Form Factors; Phys. Rov. Lett. 12, 209(1964); with P.G.0. Freund and C. R. Schumacher.

27. Consequences of Analyticity and Unitarity for Partial-wave Amplicticies; Ann. Phys. 30, 446(1964); with F. von Hippel.

28. Application of the lifethod of Homents to Partial-wave Dispersion Relations I. The Scattering of Spin Zero Particles; Ann. Phys. 30 , $4 \%(1964)$.

29. Consequences of the Strong Asymptotic Decrease of the Fixed Angle Scattering Amplitudes; Phys. Rev. 137, B177(1965).

30. Generalizations of the Pomeranchuk-Okun Theorem; Unpublished; :ith E. C. G. Sudarshan; SU-03.

31.: Ynequalities for the Solutions of Linear Integral Equations; J. of Math. Phys. Vol. 6, 1530-1533, October (1965); SU-06.

32. Criteriä for the Solubility of Dartial-wave Dispersian Relations; Ann. of Phys. 35, 209-239, November(1965); SU-12.

33. Dynamical Mixing of Spin and Unitary Spin; Not for Publication; SU-1S

34. Remarks on the Strong Asymptotic Decrease of Form Factors; Nuovo Cimento 42, ASO4(1960); SU-19.

35. Some Theorems on the Unimodular Complex Degree of Optical Coherenco; J. of Math. Physics $\underline{7}$, January (1966); with E. Wolf and C. L. Hehta; SU-33.

36. U(12) and Unitarit;; UnpubIished; SU-34.

37. Selection Rules and Lover Bounds for Scattering Anplitudes from Cure Algebras; Not for publication; with F. Nicodeai; SU-53. 
38. The Current $\Lambda$ lgebra of $S U(6)$ and Non-leptonic Hyperon Decays;

Phys. Rev. 153, 1553-1558, 25 January(1967);. with M. Gundzik and

S. Pakvasa; SU-61.

Current Algebras and Meson-Nucleon Scattering Lengths; Nuovo Cimento 44 , 1257-1261, Nugust(1966); with M. G. Gundzik and F. Nicodemi; SU-63.

40. Unitary Restrictions on Scattering Amplitudes from Dynamical Groups; Phys. Rev. 159, 1310-1320, 25 July(1967); with I. Guyk, S. Pakvasa and K. Raman; SU-73.

41. Current Algebras, Theory and Applications; Proceedings of the Boulder Conference, Boulder, Colorado, Summer 1966; with M. G. Gundzik and F. Nicodemi; SU-78.

42. Dispersion Relations; Lectures by A. P. Balachandran, Notes by M. G. Gundzik.

43. Current Commutators and Mass Extrapolations, Theory and Applications; with M. G. Gundzik and F. Nicodemi; Nuclear Physics B6, 557(1968); SU-87.

44. Current Commutators and Mass Extrapolations Theory and Applications (Revisited); with M. G. Gundzik and F. Nicodemi; SU-108.

45. Photopion Production from Nucleons; with M. G: Gundzik, P. Narayanashami and F. Nicodemi; Annals of Physics 45, 339-364, 3 December(1967); SU-114.

46. Some Consequences of the Wu-Yang Asymptotic Behavior of Form Factors; with J. J. Loeffel, Institute for Advanced Study, Princeton, N. J. preprint; Phys. Rev. 167, 1322-1334, 25 March(1968); SU-119.

47: A Simultaneous "Partial Wave" Expansion in the Mandelstam Variables Crossing Symmetry for Partial Waves with J. Nuyts; Phys. Rev. 172, $1821(1968) ; \mathrm{SU}-144$.

48. Eigenvectors for the Partial-Wave "Crossing Matrices"; with W. M. Meggs and P. Ramond; I'hys. Rev. 175, 1974, 25 November (1968); SU-160.

49. A Simultaneous Partial-Wave Expansion in the Mandelstam Variables The Group SU( 3 ); with W. J. Meggs, J. Nuyts and P. Ramond; Phys. Rev. 176, 1700-1705(1968); SU-163.

50. Generaiized Multipole Expansions; with J. Nuyts, W. J. Meggs and P. Ramo: Proceedings of the International Conference on High Energy Physics, Vienna (1968); SU-172.

51. Group Analysis of Scattering Amplitudes, The Group SU $(1,1)$; with J. Nuyts; Nuclear Physics B9, 81-88(1969); SU-183.

52. Lecture Notes on Current Algebras, Notes by W. J. Meggs; SU-166.

53. A Two Variable Expansion of the Scattering Amplitude for any Mass and Crossing Symmetry; with W. J. Meggs, J. Nuyts and P. Ramond, Phys. Rev. 187, 2080-2087, 25 November(1969); SU-192. 
54. A Two Variable Expansion of the Scattering Amplitude for any Mass and Spin and Crossing Symmetry for Partial-Waves; with it.Case and M. Modj tehedzadch; Phys. Rev. D1, 1773-1778, 15 March(1970); SU-209.

55. A Class of Dual and Factorizable N-point Functions; Phys. Rev. D1, 2770-2772, 15 May (1970); SU-210.

56. The Poincare Group, Lectures by A.P.Balachandran; Notes by D.Goswami and M.Modtehedzadeh; SU-212.

57. Integral Inequalities for the $s$ and $p$ Wave $\pi \pi$ Partial Wave Amplitudes due to Crossing Symnetry and Positivity; with W.L.Blackmon; Physics Letters 31B, 655-657, 11 Niay (1970); SU-223.

58. No-Go Theorems for Dual Models; A.P.Balachandran, L.N.Chang and P.H. Frampton; Nuovo Cimento, 1A, 545-552, February (1971); SU-232.

59. Inequalities for the $s$ and $p$ Waves $\pi \pi$ Partial ilave Amplitudes; with M.L.Blackmon; Phys. Rev. D3, 3133-3141, 15 June (1971); SU-234.

60. Inequalities for the $\pi \pi$ Partial Waves from the Properties of Class of Orthogonal Polynomials; with M.L.Blackmon; Phys. Rev. D3, 31423151 , 15 June (1971); SU-240.

61. The Crossing and Positivity Properties of Partial Waves "A Review"; Unpublished; SU-243. [Invited talk presented at the Madras Symposium on High Energy Physics, Madras, January 1971 and at the FrenchSwedish Conference on High Energy Physics, June 1971].

62. Inequalities for the Pion-Nucleon Partial Waves; with H.Case, $A$. Della Selva and S.Saito;-Nuclear Physics B31, 570 (1971); SU-249.

63. General Considerations on the Derivation of Inequalities for the s- and p-Wave Amplitudes; with M.L.Blackmon; Phys. Rev. D6, 631-639 (1972); SU-252.

64. Physical Region Constraints for $\pi-\pi$ Low Energy Parameters; with M.L.Blackmon and C.Sigaud; unpublished; SU-261.

65. Scattering of Particles with Spin: Mackey State Formulation; with J.Nilsson and L.O'Raifeartajgh; Nuclear Physics B49, 221 (1972).

66. Bounds on the Moments of Absorptive Parts of Scattering Amplitudes, with Maurice L. Blackmon and Cassio Sigaud; SU-262, Phys. Rev. D7, $3420(1973)$.

67. Relativistic Invariance and Discrete Symmetries; SU-10 (Dec. 1972) and Current Science 42, 149 (1973).

68. Dual Mode1s for Four Meson Born Terms; with H.Rupertsberger; SU-18, (March 1973) and Phys. Rev. D8, 4524 (1973). 
69. Positivity Conditions for Frampton's Dual Mode1s: with H.Rupertsberger Phys. Rey. D8, 4528 (1973).

70. A New Bound for Total Cross Section; Phys. Rev. D8, 4003 (1973).

71. Monopole Theories with Massless and Massive Gauge Fields; proceedings of the Colloquium on Group Theoretical Methods in. Physics, Marseilles, (June, 1974).

72. Monopole Theories with Massless and Massive Gauge Fields; with H.Rupertsberger and J.Schechter; Phys. Rev. D11, 2260 (1975).

73. Monopole Strings and Charmonium; with R.Ramachandran, J.Schechter, Kameshwar C. Wali and H.Rupertsberger; proceedings of the Orbis Scientiae, University of Miami, Coral Gables, Florida, January 1975.

74.: Hamiltonian Formulation of Monopole Theories with Strings; with R.Ramachandran, J.Schechter, Kameshwar C. Wali and H.Rupertsberger, Phys. Rev. D13, 354 (1976).

75. Strings, Monopoles and Meson States; with R.Ramachandran, J.Schechter, Kameshwar C.Wali and H.Rupertsberger, Phys. Rev. D13, 361 (1976).

76. Monopole Theories with Strings and their Application to Meson States; Proceedings of the Colloquium on Group Theoretical Methods in Physics, Nijmegen (1975). 


\section{Balachandran}

OTHER ACTIVITIES

Talks Given -

1. Colloquium on Group Theoretical Methods in Physics, Nijmegen, (24 June, 1975)

Monopole Theories with Strings and their Applications to Meson States.

2. Fakultat für Physik; University of Bielefeld, Bielefe1d, (1'July, 1975) Monopoles, Strings and Charmonium.

3. Institute of Theoretical Physics, Goteborg, (22 July, 1975) Report on Colloquium at Nijmegen.

4. Physics Dept., Syracuse University (27 October, 1975)

Gauge Theories, Strings, Monopoles.

Meetings Attended -

1. Colloquium on Group Theoretical Methods in Physics, Nijmegen, Holland, (June $23-27,1975$ )

2. The annual meeting of the American Association for the Advancement of Science, Boston (February 21-23, 1976).

Institutions Visited -

1. Visitor, Fakultat fur Physik, University of Bielefeld, W. Germany, (June 29-July 2, 1975).

2. Visitor, Institute of Theoretical Physics, Goteborg, (July 3-23, 1975$)$.

3. Visitor, Center for Theoretical Studies, Indian Institute of Science, Bangalore, (July 28-August 19, 1975).

Referee for

The Physical Review

The Physical Review Letters 


\section{Bibliography of Stephen Biaha}

1. Continuum Contributions to the Pion Radius, Phys. Rev. 178,2167 (1969).

2. Character Analysis of $\mathrm{U}(\mathrm{N})$ and $\mathrm{SU}(\mathrm{N})$; J.Math. Phys. 10, 2156 (1969).

3. The Deep Inelastic e-p Structure Functions in a Ladder Model with Spin 1/2 Nucleons, Phys. Rev. D3, 510 (1971).

4. The Inelastic e-p Structure Functions in a Gluon Mode1; Phys. Lett. $40 \mathrm{~B}, 501$ (1972).

5. A Universal Curve for Meson-Nucleon Elastic Scattering; Phys. Lett. $42 \mathrm{~B}, 435$ (1972). (with U.Sukhatme and W.J.Pardee).

6. Consistency of New Meson-Nucleon Elastic Scattering Data with a Previously Conjectured Universal Curve; with U.Sukhatme, Phys. Rev. D8, 4221 (1973).

7. An Approximate Calculation of the Eigenvalue Function in Massless Quantum Electrodynamics, Phys. Rev. D9, 2246 (1974).

8. A Model for Pion Condensation; Nuovo Cimento 25A, 252 (1975).

9. Action at a Distance and Hadron Binding; Phys. Lett. 56B, 373 (1975).

10. Towards a Fie1d Theory of Hadron Binding; Phys. Rev. D10, 4268 (1974).

11. A Second quantized Non-Abelian Field Theory for Hadrons with Quark Confinement and Scaling Deep Inelastic Structure Functions; Phys. Rev. D11, 2921 (1975).

12. Relativistic Bound State Models with Free Constituent Motion at Spacelike Distances; Phys. Rev. D12, 3921 (1975).

13. Quantization Rules for Point Singularities in Superfluid ${ }^{3}$ He and Liquid Crystals,; Phys. Rev. Lett. 30, 874 (1976). C00-65

14. An Experimentally Accessible Analog of the Magnetic Monopole in ${ }^{3} \mathrm{He}-\mathrm{A}$; (submitted for publication). April 1976 C00-69

15. Yang-Mills Approach to the Superfluid ${ }^{3}$ He Free Energy; (submitted for publication). April 1976 C00-70

16. Quantum Gravity and Quark Confinememt; (submitted for publication) 5/76. C00-68

17. A Possible Relation Between the Confinement of quarks and Renormalizability of quantum Gravity; (to be submitted for publication) 5/76 $\mathrm{C} 00-73$

18. On Embedding a Classical Field Theory in a Quantum Field Theory; (to be submitted for publication) $\mathrm{C} 00-74$

19. Yang-Mills Generalization of Weyl's Unified Field Theory.(in preparation) $\mathrm{C} 00-75$ 
Blaha

OTHER ACTIVITIES

Talks Given:

Williams College, Williamstown, Mass. (February 1976)

Relativistic Bound State Quark Models

Syracuse University (Sept. 1975 - May 1976)

Series of talks on Topology of Magnetic Monopoles

Two talks on Anomalous Lepton Production in $\mathrm{e}^{+} \mathrm{e}^{-}$Annihilation. Three Seminars and Colloquia on my research interests.

Meetings Attended:

Annual APS meeting in New York, N.Y. February 2-6, 1976

Other

Member APS

Member Sigma Xi

Essay on "Guantum Gravity and Quark Confinement" submitted to the Gravity Research Foundation, received honorable mention and was considered by the judges to be of outstanding quality. 
Bibliography of Steven R. Borchardt

1. Renormalizability, Gauge Invariance and $\nu_{e} \nu_{\mu}$-Scattering in Weinberg-Salam Mode1; with K.T.Mahanthappa; Nucl. Phys. B65, 445 (1973).

2. Spectral Function Sum Rules in Asymptotically Free Theories; with V.S.Mathur; Phys. Rev. D9, 2371 (1974).

3. A Possible Explanation of the New Resonance in $\dot{e}^{+} \mathrm{e}^{-}$Annihilation; with V.S.Mathur and S.Okubo; Phys. Rev. Letters 34, 38 (1975).

4. SU(4) Symmetry and the Possible Existence of New Hadrons; with S. Okubo and V.S.Mathur; Phys. Rev. Letters 34, 236 (1975).

5. SU(4) Symmetry and the New Resonances; with V.S.Mathur and S.Okubo; Phys. Rev. D11; 2572.(1975).

6. Weak Decay Modes of Charmed Mesons; with V.S Mathur (University of Rochester preprint) accepted for publication in Phys. Rev. Letters. (1976)

7. Weak Decays of Charmed Hadrons in Vector Dominance Mode 1; with V.S.Mathur in preparation. (1976)

Meetings attended:

1. International Conference of the Production of Particles with new Quantum numbers, University of Wisconsin, Madison, Wisc. 4/76

2. APS meeting, Washington, D.C., Apri1 26-29, 1976.

Talks given:

1. University of Rochester, New York

Charmed Meson Decays. (March 1976)

2. Syracuse University, New York

Charmed Meson Decays, (April 1976) 


\section{Bibliography of Robert F.Cahalan}

1. Cluster Expansion in a Renormalizable Theory: The Elastic Form Factor; Phys. Rev. D5, 2999 (1972).

2. $Z^{\circ}$ Photoproduction at High Energy; Phys. Rev. D9, 257 (1974).

3. The $Z^{\circ}$-effect in Lepton-Pair Photoproduction; with K.0.Mikaelian, Phys. Rev. D10, 3769 (1974).

4. Spontaneous Symmetry Breakdown and Regge Intercepts; with P.H.Frampton; Physics Letters 50B, 475 (1974).

5. The Elastic Form Factor in a Non-Abelian Gauge Theory; with P.M.Fishbane; University of Virginia preprint.(Nov. 1974).

6. Asymptotic Freedom and the "Absence" of Vector-gluion Exchange in Wide-angle Hadronic Collisions; with K.A.Geer, J.Kogut and L.Susskind; Phys. Rev. D11, 1199 (1975) .

7. Construction of Planar Graphs: with D.Knight; Syracuse University preprint SU-57. (1976)

\section{other Activities}

Exchange Scientist, National Academy of Science and Polska Akademia Nauk, Institute of Theoretical Physics, Warsaw, Poland, March to Ju1y 31, 1975 .

Meetings attended:

Conference on Hadrons; Zakopane, Poland. (June 1975)

Conference on Neutrinos, Balaton, Hungary. (June 1975)

APS Annual Meeting, New York, N.Y. (February 2-6, 1976)

Member APS 
Biblography of Sung-Sheng Chang

1. Effective Lagrangian Density in Gauge Symmetry; C00-67, submitted to Physical Review D, March 1976.

2. Exact Integration on Feynman Path Integrals; C0O-72, submitted to Phys. Rev. D. April 1976.

3. Current Algebra Evaluation of Pion Pair Production in Two-photon Processes; with R. Arnowitt; submitted to. Phys. Rev. D. April, 1976 .

Meetings attended:

Northeastern University Conference, Boston, Mass.

Talks Given

Syracuse University

Exact Integration on Feynman Path Integrals. March 1976 (seminar) 
Threc Photon Decay of Positronium ${ }^{1} \mathrm{~S}$ state as a Test of Charge Conjugation Invariance; Phrs. Rev. 132, $841(1963)$. 2. Possible Existence of a Boson Isocuplet; Phys. Rev. 135, B219(1064);
with B. Lee and S. Okubo.

3. Prediction of a $\pi n$ Resonance; Phys. Rev. 135, B1060(1964); with

S. Okubo.

4. Pcrmutation Symietry and a Derivation of Unitary Symmetry; Ann. Phys. 32, 124(1965); with Y. Ueda and S. Okubo.

5. Higher Syminetry Grouns with Parity lixing; Prog. Theor. Pliys. Supplanant 1965,57 ; with R. Marshak, S. Okubo and J. Nojtcszik.

6. The Strangeness Changing Axial Vector Coupling Constart; Fhys. Letlers 1.9, 56(1965); with L. Pandit.

7. Weak Currents in Broken U(3) x U(3); Phys. Rev. 144, 1938(1965); with Y. Ueda.

8. Nonleptonic Decays of Hyperons; Phys. Rev. Letters 16, 330(1966); with Y. Hara and $Y$. Nambu.

9. Nonlcptonic Decays of Hyperons in Broken SU(3) $x$ SU(3); Phys. Rev. I4S. $1424(1966)$; with Y. Ueda. 10. Neak Intcraction Universaiity and Octet Dominance; Phys. Rev. Letters
... 16; $1022(1956)$; with Y. Chiu.

11. Theory of Nonleptonic Hypcron Decays; Phys. Rev: 150, 1201(1960); with $Y$. Chili and $Y$. Ueda.

12. Nass Splitting in the Current-Current Picture; Nuovo Cimento 47, 214 (1967); with Y. Chiu.

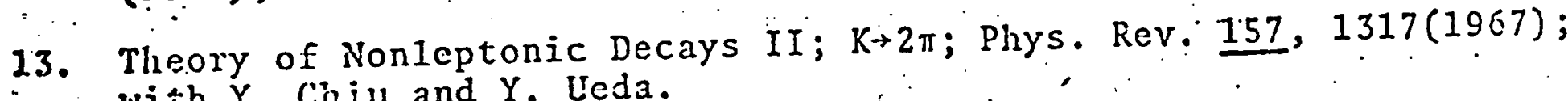
with $Y$. Cniu and $Y$. Leda.

14. Meson-Mcson Scattering in the Quark Nodel and the Adler Sum Kules; N. C. Letters 66, 5+S(1960); with Y. Chiu.

15. Algebra of Currents and Three pion Decay Hodes of $n$ and $K$ Mesons; Phys. Rev. 151, 1612(196i); with Y. Chiu and Y. Ueda. 16. CP Violation in a Current-Current Mode1; Phys. Rev. 167, 1345(1963);
with Y. Coiu; SU-136.

Symmetry Restrictions on $\mathrm{K}-2 \pi$ Amplitudes; Phys. Rev. $161,1660(106 ?)$. 18. Asymptotic Chiral Symmetry; Phys. Rev. Letters 19, 276(1967); with G. Venturi. 
19. Final State Interaction Effects in the Algebra-of-Currents Approach to the Three-Pion Decay Hodes of $n$ and $K$ Mesons; Phys. Rev. 161 , 1612(1967); with Y.T.Chiu and Y.Ueda.

20. Phenomenological Chiral Model for Nonleptonic Hyperon Decays; phys. Rev. 174, 1829 (1969); SU-148.

21. $\mathrm{K}^{+}$-Nucleon Scattering in an Effective Chiral Lagrangian Mode1; Phys. Rev. 177, 2311(1969); SU-159.

22. Baryon Excitation Form Factors and Asymptotic Chiral Symmetry; Phys. Rev. 177, 2300 (1969); SU-162.

23. Note on the Gauge Field Chiral Lagrangian; Phys, Rev. 188, 2184 (1969); with Y.Deda; SU-194.

24. $\Omega^{-}$-Decay; with D.N.Goswami; Phys. Rev. D1, 290(1970); with D.N.Goswami; SU-199.

25. Spontaneous Breakdown of Weak and Electromagnetic Interaction Symunetry; Phys. Rev. D2, 736(1970).

26. Some New Results on Symmetry breaking in a Chiral Model; (unpublished); with Y.Ueda; SU-228.

27. Electronagnetic Perturbation of the Pseudoscalar Mass Spectrum; Phys. Rev. D3, 176(1971); with Y.Ueda; SU-229. 28. Symmetry Breaking and Spin Zero Mass Spectrum; Phys. Rev. D3, 168
(1971); with Y.Ueda; SU-230.

29. Van Hove Model and Nonleptonic Hiyperon Decays; Phys, Rev. D3, 2128 (1971); with D.N.Goswami and Y.Ueda; SU-238.

30. General Treatment of Chiral and Scale Breaking in the SU(3) Sigma Model; Phys. Rev. D3, 2874(1971); with Y.Ueda; SU 241 .

31. $\Delta I=I$ Mass Differences and $n \rightarrow 3 \pi$ Decay; with Y.Ueda; Rhys. Rev. DI, 733(1971); SU-245.

32. CP Violation and Chiral Symmetry; with Y.Ueda; Phys. Rev. D5, 2821, (1972): SU-253.

33. Symmetry Breaking and the Pionic Decays of $K$ Mesons; with D.Goswami and Y.Ueda; Phys. Rev. D5, 2276(1972); SU-256.

34. A Possible Origin for Symmetry Breaking; with Y.Ueda; Phys. Rev. D5, 2846(1972), SU-257. 
35. Unified Weak-Electromagnetic Gauge Schemes Based on the Three Dimensional Unitary Group; with Y. Ueda; Phys. Rev. D8, 484 (1973).

36. High Energy Behaviour of Gauge Theory Tree Graphs; with Y.Ueda, Phys. Rev. D7, 3119(1973).

37. $\eta+3 \pi$ in a Renormalizable SU(3) Sigma Model; with W.Hudna11; Phys. Rev. D9, 2111 (1973).

38. Phenomenological Dynamics for CP Violation; with M.Singer; Phys. Rev. D8, 3866(1973).

39. Higgs Mesons and High Energy Behaviour; with Y.Ueda; Lettere al Nuovo Cimento $\underline{8}, 991(1973)$.

40. Spontaneous "Cabibbo" Suppression; with M.Singer; Phys. Rev. D6, 1769 (1974).

41. Monopole Theories with Massless and Massive Gauge Fields, with A.P.Balachandran and H.Rupertsberger, Phys. Rev. D1I, 2260 (1975).

42. Semi-Leptonic Neutral Current Decays; with M.Singer. II Nuovo Cimento 26A, 117 (1975).

43. Monopole Strings and Charmonium; with A.P.Balachandran, R. Ramachandran, H. Rupertsberger and Kameshwar C.Wali. Proceedings of the Orbis Scientiae, University of Miami, Coral Gables, Florida, January 1975.

44. Hamiltonian Formulation of Monopole Theories with Strings; with A.P.Balachandran, R. Ramachandran, H. Rupertsberger and K. C. Wali, Phys. Rev. D13, 354 (1976):

45. Strings, Monopoles, and Meson States; with A.P.Balachandran, R. Ramachandran, H.Rupertsberger and K.C.Wali. Phys. Rev. D13, 361 (1976).

46. SU(4) Sigma Mode1; with M.Singer, Phys. Rev. D12, 2781 (1975).

47. Possible Enhancement of the Leptonic Decays of Charmed Pseudoscalars; with J.Kandaswamy and M.Singer, Phys. Rev. D, to be published.

48. Yang-Mills Particle in 't Hooft's Field; submitted for publication. 


\section{Meetings attended}

Northeastern University conference, Boston, Mass. September 25-28, 1975 .

Other

Member APS 
Bibliography of K. C. Hali

1. Theory of Photoproduction of Pions from Nucleons; J. Enoch, R.G.Sachs and K.C.Wali, Phys. Rev. 10S, No.2, 433-455, (October 15, 1957).

2. Elcctronagnetic Form Factors of the Nucleon; F.J. Ernst, R.G.Sachs, and K.C.Hali, Phys. Rev. 119, No.3, 1105-1114, (August 1, 1960).

3. Pion-Pion Interactions in $\tau$ and $\tau$ ' Decays; R.G.Sawyer and K.C.Wali; Phys. Rev. 119, No.4, 1429-1435, (Nugust 15, 1960). .

4. Tcsts for $\Delta T=1 / 2$ in $K^{+} \rightarrow 3 \pi$ and $K_{2}^{\circ} \rightarrow 3 \pi$ Decay Modes; R. G. Sawyer and K.C.WaIi, Nuovo Cimento, Series X $\mathrm{X}^{2}$, 938-945, (September 16, 1960).

5. Scattering Matrix Approach to the $\Lambda-\pi$ Resonances; G. Feldman, T. Iulton and K.C.Wali, Phys. Rev. Letters 6 , 664 (1961).

6. Some Consequences of the Decay Modes and Production of the New Heavy Bosons; G. Feldman, T. Fulton and K.C.Wali, Nuovo Cimento Series $X$ 24, 278-289(1962).

7. Effect of High Energy Limits on the Nucleon Form Factors; K.C.Wali; Nuovo Cimento, Series X 25, 912-922(1962).

8. $3 \pi^{0}$ to $\pi^{+} \pi^{-} \pi^{0}$ Branching Ratio of a $0^{-+} \mathrm{n}$ Meson; K.C. Hail, Phys. Rev. Letters 9, (August 1, 1962).

9. Coupled Channel Approach to $J=3^{+} / 2$ Resonances in the Unitary
Symmetry Model; A.W. Martin and K.C.Wali, Phys.Rev. $130,2455-2467$ (1962).

10. Meson-Baryon Resonances in the Octet Model; A.W.Martin and K.C.Hali, Nuovo Cimento 31, 1324 (1964).

11. Implications of a Possible $\mathrm{J}^{P}=3+/ 2$ Octet; E.Johnson, R. F. Sawyer and K.C.Wali, Phys. Rev. Letters 13, $141(1964)$.

12. A Dynamical Model for a $J^{P}=3+/ 2$ Nonuplet; J.J.Brehm and K.C.llali, Physics Letters 11, $355(1964)$.

13. Breaking of $S U(3)$ in the $3+/ 2$ Meson-Baryon Decuplet; K.C.Wali and R.L. Varnock, Phys. Rev. 135, B1358(1965).

14. A Phenomenological Approach to SU(6) Theory; B.Sakita and K.C.Wali, Phys. Rev. Letters 14, $11(1965)$.

15. A Relativistic Formulation of SU(6) Symmetry Scheme; B. Sakita and K.C.Wali; Phys. Rev. 139, B1355(1965). 
Wali

16. Linear and Non-Lincar Mass Difference Effects in a Model of the Baryon Multiplets; F.J.Erust, R.Warnock and K.C.liali, Phys. Rev. J.41, $1354(1966)$.

17. Effects of $\mathbf{C}$ Nonconservation in Eta Decay into Pions; D.Griffiths, R.F.Sawyer and K.C.Hali; (Unpublished).

18. A Model for Low Energy Meson-Baryon.Scattering; Gary R. Goldstein and K.C.Wali; Phys. Rev. 155, 1762 (1967).

19. Sum Rules for Baryon Resonance Widths; Bunji Sakita and K.C.Wali, Phys. Rev. Letters 18, 31(1967).

20. Effects of Continuum Contributions to Vector Meson Propagators; Michael T.Vaughn, Haurice L.Blackmon and K.C.Wali (submitted io the Vienna Conference 1968).

21. Continuum Contributions to Vector-Meson Decays; Michael T.Vaughn and K.C.Wali; Phys. Rev. Letters 21, $938(1968)$.

22. Continuum Contributions to Vector Meson Decays II. Broken $U_{3}$ Symmetry; Michael T.Vaughn and K.C.Wali; Phys.Rev.177, 2199- 2205 (1969).

23. The Vencziano Model for $\pi \pi \rightarrow \pi S$ where $S$ has Arbitrary Spin and Parity; C.J.Goebel, Maurice L.Blackmon and K.C.Wali; Phys.Rev.182, $1487-1491(1969)$.

24. Pion-Nucleon Scattering with Veneziano Terms; S.Fenster and K.C..liali; Pliys. Rev. DI, 1409-1416(1970).

25. A Veneziano Model for the Reaction $\pi^{-} p \rightarrow n n$; Maurice L.Blacknon and K.C.Wali; Phys. Rev. D2, 258-261(1970); SU-215.

26. Implications of Local Duality in Meson Decuplet Scattering; M.J.King and K.C.Wali; Phys. Rev. Letters 24, 1460-1463(1970); SU-224.

27. Implications of Local Duality in a Set of Coupled Reactions; M.J.King and K.C.Wali; Phys. Rev. D3, 1602(1971); SU-235.

28. s-Channel Helicity Conservation Constraints on $t$-Channel Exchange; M.L.Blackmon, N.J.King and K.C.Hali; Phys. Rev. 559-569(1971); SU- 242 .

29. Restrictions on the Angular Momentum of $t$-Channel Exchange from s-Channel Helicity Conscrvation; M.L.Blackmon, M.J.King and K.C.liaii; Physics Letters 35B, 44(1971); SU-248.

30. Consequences of Local Duality in Ki Scattering; M.J.King, P.Shah and.K.C.Wali, Phys:Rev. D6, 229-237, I July 1972; SU-258. 
31. Properties of Polarization Density Matrix in Regge Pole Mode 1s; with M.Doce1, P.Mery, L.Miche1, Pierre Minnaert and K.C.Wali; printed-Institue des Hautes Etudes Scientifiques, Bures-sur-Yvette, France. Phys. Rev.

32. Regge Slope Expansion of Meson Born Amplitudes; with P.Frampton; Phys. Rev. D8, 1881 (1973).

33. Monopole Strings and Charmonium; with A.P.Balachandran, R. Ramachandran, H.Rupertsberger and J.Schechter. Proceedings of the Orbis Scientiae, University of Miami, Coral Gables, Florida, January 1975 .

34. SU(4) Clebsch-Gordan Coefficients; with V.RabI and G.Campbe11,Jr. Journal of Math. Phys. 16, 2494 (1975).

35. Resonance-sum Model for Reggeization in Scattering of Particles with Arbitrary Spin; with L.Durand and M.J.King; Phys. Rev. D13, 1409 (1976).

36. Hamiltonian Formulation of Monopole Theories with Strings; with A.P.Balachandran, R. Ramachandran, H.Rupertsberger and J.Schechter; Phys. Rev. D13, 354 (1976).

37. Strings, Monopoles, and Meson States; with A.P.Balachandran, R.Ramachandran, H.Rupertsberger and J.Schechter. Phys. Rev. D13, 361 (1976).

38. Current Status of Baryon Spectroscopy; talk published in "New Direction of Hadron Spectroscopy" proceedings of the International symposium,. Argonne, July 1975 . 


\section{OTHER ACTIVITIES}

\section{Meetings Attended}

1. International Symposium, New Direction of Hadron Spectroscopy, Argonne National Laboratory, Argonne, I11. (July 7-10, 1975).

2. Sabbatical for the fall semester 1975:

Institut des Hautes Etudes Scientifiques, Bures-sur-Yvette, France.

3. Orbis Scientiae Conference, University of Miami, Coral Gables, Florida. (January 18-21, 1976)

4. Fermi National Accelerator Laboratory, Batavia, Illinois, for research study, (March 8-12, 1976)

5. Brookhaven National Laboratory, Upton, N.Y., Publication Committee Meeting APS, April 15, 1976.

6. International Conference on the Production of Particles with new Quantum numbers, University of Wisconsin, Madison, Wisc.(Apri1 2124;: 1976)

7. APS Meeting, Washington, D.C., Apri1 26-29, 1976.

Talks Given

Argonne International Symposium, July 1975

Current Status of Baryon Spectroscopy

Institute for Theoretical Physics, Nijmegen, The Netherlands, Aug. 1975 Current Status of Baryon Spectroscopy

Institut des Hautes Etudes Scientifiques, Bures-sur-Yvette, France, $10 / 75$ Monopoles, Strings and Charmonium

CNRS, Marseille, France, November 1975

Non-Ableian Monopole The ories.

University of Bordeaux, France November 1975

Regge Formula for Arbitrary Spin Particle Scattering 
Talks given cont'd

University of Delhi, India, November 1975

Monopoles, Strings and Charmonium (colloquim, 11/26)

't Hooft Monopoles and Possible Generalizations (seminar, 11/27).

Military Academy School, Dehradun, India Dec. 1975

Current Ideas in Modern Physics

Indian Institute of Technology, Kanpur, India, Dec.1975

Concerning 't Hooft Monopoles

Monopoles, Strings and Charmonium (seminars)

Tata Institute of Fundamental Research, Bombay, India Dec.1975

A Resonance-sum Mode 1 for Reggeization (seminar)

Monopoles, Strings and Charmonium (colloquim)

Karnatak University, Dharwar, India Dec. 1975

Hadron Spectroscopy-I, Descriptive (seminars)

Hadron Spectroscopy-II, Recent Theoretical Attempts to Understand it.

Present Status of our Understanding of the Nature of Matter. ( A Silver Jubilee Public Lecture)

Syracuse University, May 1976

Report on the Wisconsin and Washington meetings. (seminar)

Other

Fellow APS

Member RESA

Referee for - Physical Review Physical Review Letters

AEC Proposals

NSF Proposals 
$m-1$

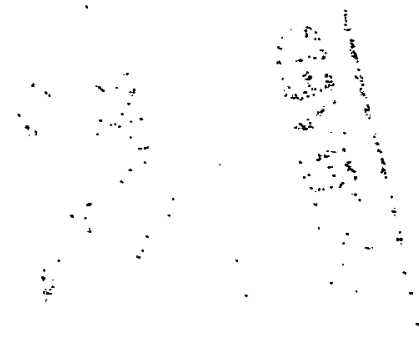

$\therefore$

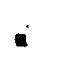

5

$=8$
.

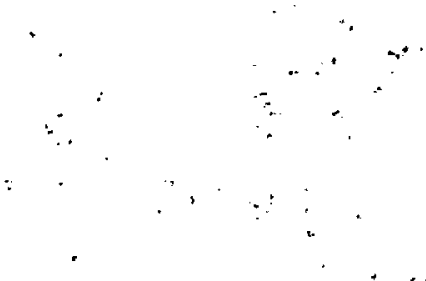

$\therefore$ 